\title{
La sopravvivenza dell'accesso vascolare nei pazienti emodializzati
}

\author{
Giuseppe Bacchini
}

\author{
Dipartimento di Nefrologia e Dialisi, Ospedale "A. Manzoni”, Lecco
}

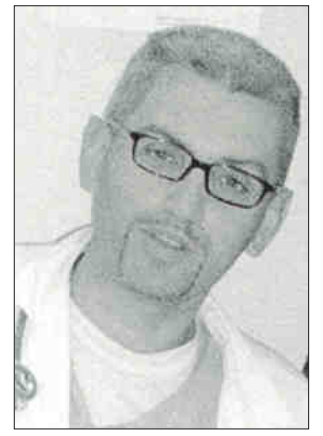

scolare ideale per
il paziente in trat-
tamento emodia-
litico è quello
che consente ade-
guati flussi e ri-
mane pervio, con
una bassa inci-
denza di complicanze, più a lungo. Nessun accesso vascolare per ora è stato in grado di soddisfare a tutti questi criteri. La recente pubblicazione del National Kidney Foundation Dialysis Outcome Quality Initiative (DOQI) guidelines (1) sull'accesso vascolare, riconosce la superiorità dell'accesso vascolare con vasi nativi $(2,3)$, consigliandone quindi un approccio più aggressivo nel paziente emodializzato. In Italia, la fistola arterovenosa (FAV) rappresenta l'accesso vascolare d'elezione per il trattamento emodialitico (4), mentre negli Stati Uniti d'America l'accesso vascolare protesico (AVP) con polytetrafluoroethylene (PTFE), rappresenta spesso la scelta più frequente .

\section{Il nefrologo interventista}

Dai dati recenti della letteratura appare evidente come sia nella popolazione incidente che prevalente, in Italia principalmente e più in generale in Europa, più dell' $80 \%$ dei pazienti ha come accesso vascolare una fistola con vasi nativi (4). In questo studio viene altresì messo in luce come in Italia il $90 \%$ degli interventi di allestimento dell'accesso vascolare vengono effettuati dai nefrologi, senza l'intervento di chirurghi vascolari, se non per gli interventi più complessi. Questi dati emergevano anche da una recente pubblicazione (5). Non solo quindi gli accessi vengono allestiti dai nefrologi, ma si evidenzia una ottima sopravvivenza degli accessi vascolari con i vasi nativi sia rispetto agli accessi in protesi che rispetto all'accesso vascolare con vasi nativi confezionati negli USA (5). Dati praticamente sovrapponibili come sopravvivenza degli accessi vascolari nativi e in protesi, sono stati recentemente pubblicati anche da uno studio italiano (6). A questo punto appare lecito chiedersi l'utilità di un protocollo di sorveglianza degli accessi vascolari sia per le fistole native che per gli accessi in protesi. Secondo la più recente letteratura $(1,6)$ l'alta sopravvivenza degli accessi vascolari con i vasi nativi, giustifica la presenza di un protocollo di sorveglianza molto snello, basato sulla raccolta di alcuni dati come la pressione venosa statica e il ricircolo. Solo nel caso di parametri alterati, si sottopone il paziente a un controllo ecocolordoppler dell'accesso vascolare. Il protocollo di sorveglianza per gli accessi vascolari in protesi deve invece prevedere la misura con cadenza mensile della portata della protesi, a cui far seguire in caso di riduzione in percentuale superiore al $35 \%$ (7), un controllo ecocolordoppler.

Nel caso di riscontro di stenosi emodinamicamente significativa (8), si deve nella stessa seduta procedere a una angioplastica (PTA) della zona stenotica, sempre sotto guida ecografica.

Tale metodica (9) consente, da una parte una risoluzione sia funzionale che morfologica della lesione e dall'altra, evita al paziente l'esposizione alla radiazione e al mezzo di contrasto; non da ultimo viene effettuata da 


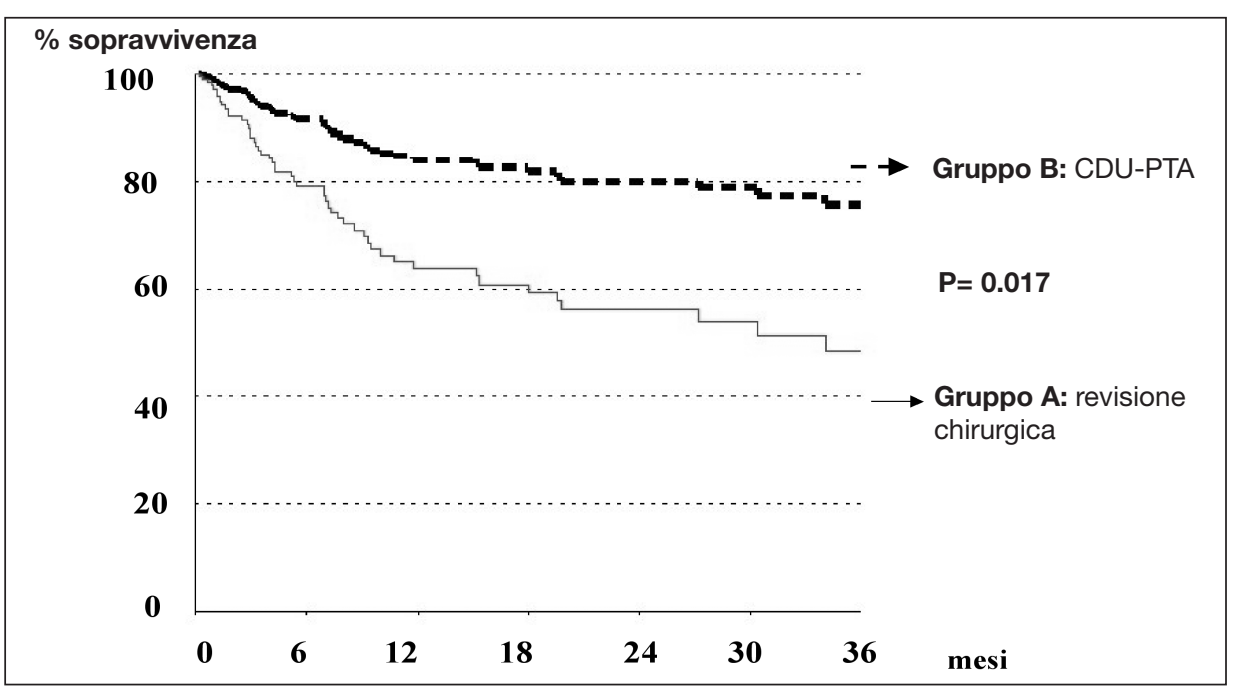

Fig. 1 - Gruppo B: pazienti con effettuazione di PTA sotto guida ecografica; Gruppo A: pazienti con solo approccio chirurgico.

un'équipe completamente nefrologica, con un risparmio, rispetto alla classica metodica sotto guida angiografica, del $20 \%$.

L'utilità delle metodiche di radiologia interventistica sia sulle fistole che sugli accessi vascolari in protesi sono state molto bene evidenziate da un recente articolo del gruppo di Turmel Rodrigues (10). La nuova metodica di radiologia interventistica ecoguidata, in un recente lavoro (11), ha evidenziato come da sola, rispetto all'approccio solamente chirurgico, sia l'unico evento in grado di determinare un miglioramento statisticamente significativo (Fig. 1) della sopravvivenza degli accessi vascolari $(\mathrm{p}=0.017)$, con una riduzione del $64 \%$ del rischio di trombosi.

Queste nuove metodiche di radiologia interventistica ecoguidata, consentono anche la trombectomia, per via percutanea, mediante metodo meccanico, di fistole e di accessi protesici. Si ottiene quindi un minor utilizzo della sala operatoria, con l'effettuazione di una metodica in regime di Day Surgery, completamente gestita dal personale nefrologico. Solo un $10 \%$ degli interventi di mantenimento degli accessi vascolari rimangono quindi di esclusiva pertinenza chirurgica. Tutto ciò con un evidente vantaggio soprattutto per il paziente, non più costretto a ricoveri e interventi chirurgici più o meno complicati, che rendono difficile la gestione dell'accesso vascolare.

Alla luce di queste nuove metodiche e degli ottimi risultati in termini di sopravvivenza degli accessi vascolari sia con vasi nativi (6), che in seconda battuta con l'interposizione di un pontaggio (10), il catetere centrale a permanenza, deve rimanare, come lo è già in Italia (4), la terza scelta nella storia dell'accesso vascolare del paziente emodializzato.

Tutto questo bagaglio culturale e professionale del nefrologo nella gestione degli accessi vascolari va quindi valorizzato sempre di più, con la creazione di équipe di nefrologia interventistica ad hoc.

\section{BIBLIOGRAFIA}

1. NKF-DOQI clinical practice guidelines for vascular access. National Kidney Foundation. Am J Kidney Dis 1997; 30 (Suppl.): S152-91.

2. Hirth RA, Turenne MN, Woods JD, et al. Predictors of type of vascular access in hemodialysis patients. JAMA 1996; 276: 1303-8.

3. Rohr MS, Browder W, Frentz
GD. Arteriovenous fistulas for long term dialysis. Factor that influence fistula survival. Arch Surg 1978; 113: $153-5$.

4. Ronald L. Pisoni, Young E, et al. Vascular use in Europe and the United States: results from DOPPS. Kidney Int 2001; 61: 305-15.

5. Bonucchi D, D'Amelio A, Capelli $\mathrm{G}$, et al. Management of vascular access for dialysis: An italian survey. Nephrol Dial Transplant 1999; 14: 2116-8.

6. Bacchini G, Andrulli S, Pontoriero G, La Milia V, Locatelli F. Sopravvivenza degli accessi vascolari nativi e protesici nei pazienti emodializzati. Giorn Italiano Nefrologia $2000 ; 17,4: 369-74$.

7. Neyra NR, Ikizler TA, May RE, Himmelfarb J, Schulman G, Shyr Y, Hakim RM. Change in access blood flow over time predicts vascular access thrombosis. Kidney Int 1998; 54: 1714-9.

8. Berguer R, Hwang NHC. Critical arterial stenosis: a theoretical and experimental solution. Ann Surg 1974; 180: 39-50.

9. Bacchini G, Capello A, La Milia V, Andrulli S, Locatelli F. Color doppler ultrasonography imaging to guide transluminal angioplasty of venous stenosis. Kidney Int 2000; 58: 1810-3.

10. Turmel-Rodrigues L, Pengloan J, Hervé R, et al. Treatment of failed native arteriovenous fistulae for hemodialysis by interventional radiology. Kidney Int 2000; 57: 1124-40.

11. Bacchini G, Andrulli S, La Milia V, Locatelli F. Surveillance protocol with color doppler ultrasonography imaging to guide transluminal angioplasty of venous stenosis in vascular access grafts of hemodialysis patients. J AM Soc Nephrol 2001; 12: A1445.

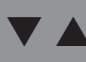

